\title{
Experimental Results of Acoustic Emission Attenuation Due to Wave Propagation in Composites
}

\author{
Sebastian F. Wirtz ${ }^{1}$, Stefan Bach ${ }^{2}$, Dirk Söffker ${ }^{3}$ \\ 1,2,3 Chair of Dynamics and Control, University of Duisburg-Essen, 47057 Duisburg, Germany \\ sebastian.wirtz@uni-due.de \\ stefan.bach@stud.uni-due.de \\ soeffker@uni-due.de
}

\begin{abstract}
Recently, acoustic emission-based damage classification schemes gained attention for health monitoring of composites. Here, the reliable detection of different micromechanical damage mechanisms is important because of the adverse effect on fatigue life. It is well known that classical parameters obtained from acoustic emission measurements in time domain are strongly dependent on the propagation path and testing conditions. However, signal attenuation, which can be observed due to geometric spreading, material-related damping, and dispersion, is typically neglected. Therefore, it is generally assumed that frequency domain features are reliable descriptors of damage due to invariance of peak frequencies to the propagation path. Based on this assumption, several data-driven approaches for damage detection are developed. However, in contrast to metallic materials, where low attenuation is observed, acoustic emission signals are strongly attenuated in polymer matrix composites due to viscoelastic behavior of the matrix. For instance, it is reported in literature that at high frequencies most of the acoustic emission signal energy is attenuated after a propagation distance of $250 \mathrm{~mm}$. Therefore, new experimental results of acoustic emission attenuation in composites are presented in this paper. Particular focus is placed on the frequency dependence of acoustic emission attenuation and the effect of different loading conditions. The specimens are manufactured from aerospace material. Carbon fiber reinforced polymer plates are used as a typical specimen geometry. Different acoustic emission sources are considered and the related attenuation coefficients are determined. Furthermore, full waveform data are analyzed in time and time-frequency domain using wavelet transform. From the experimental results it can be concluded that consideration of wave propagation-related signal attenuation is important
\end{abstract}

Sebastian Felix Wirtz et al. This is an open-access article distributed under the terms of the Creative Commons Attribution 3.0 United States License, which permits unrestricted use, distribution, and reproduction in any medium, provided the original author and source are credited. for the interpretation of acoustic emission measurements for health monitoring of composites. Consequently, the impact on the detectability of different physical damage mechanisms using data-driven classification approaches has to be considered.

\section{INTRODUCTION}

Today, composites are widely used in different applications due to their good mechanical properties. However, their use is currently limited by a lack of ductility and several micromechanical damage mechanisms, which adversely effect the fatigue behavior of these materials. Therefore, the reliable detection and characterization of damage is crucial for health monitoring of composite structures. In this context, Acoustic Emission (AE) measurements are widely used. The passive acquisition of ultrasound stress waves, which propagate in a structure due to damage, allows in-situ detection and localization of damage in large structures with only few sensors using suitable signal processing techniques.

In composite material, characteristic $\mathrm{AE}$ waveforms are observed, which can be attributed to different micro-mechanical damage mechanisms. For instance, delamination is characterized by dominant out-of-plane motion in a composite plate, whereas fiber breakage excites primarily in-plane source motion (Crivelli et al., 2015). In plate specimens, these waveforms propagate usually as fundamental symmetric and asymmetric Lamb wave modes $S_{0}$ and $A_{0}$. Different $\mathrm{AE}$ waveform features can be exploited to characterize the underlying micro-mechanical damage mechanism. However, according to (Johnson \& Gudmundson, 2000), classification of AE measurements in time domain using classical waveform parameters is considered as difficult due to propagation effects such as amplitude attenuation. According to (Ni \& Iwamoto, 2002), peak frequencies are less sensitive to the propagation path and hence more effective for monitoring of composite materials. Therefore, frequency and time- 
frequency domain analyses are excessively used for characterization of $\mathrm{AE}$ waveforms.

As reported in literature, different data-driven classification schemes can be implemented for automated characterization of $\mathrm{AE}$ waveforms. Wavelet transform is used by (Marec, Thomas, \& El Guerjouma, 2008) to define new features which can be used to distinguish between matrix cracking and debonding. Furthermore, (Gutkin et al., 2011) studied AE obtained from tensile tests with different specimens using unsupervised techniques. According to the results, clusters agree with patterns in the peak frequency distribution. In contrast, (Hamdi et al., 2013) used Hilbert Huang Transform (HHT) to extract $\mathrm{AE}$ features in time-frequency domain. Effectiveness of the approach is demonstrated using k-means clustering. Similarly, (Nazmdar Shahri, Yousefi, Fotouhi, \& Ahmadi Najfabadi, 2016) developed a signal processing technique based on HHT to characterize different damage mechanisms. A methodology based on multivariable analysis of wavelet coefficients is developed by (Baccar \& Söffker, 2017) to improve the classification and distinguishability of four different damage mechanisms. Similarly, time-frequency domain transforms including wavelet transform and Choi-Williams transform are used by (Beheshtizadeh, Mostafapour, \& Davoodi, 2019) to identify characteristic frequencies of different damage mechanisms in composite materials. Scattering of the classification reliability among different loading conditions using a supervised data-driven method is reported by (Wirtz, Beganovic, \& Söffker, 2019).

Another approach, which exploits theoretical knowledge about wave propagation, is modal AE. Here, amplitude ratios of the two fundamental Lamb wave modes are evaluated to characterize the underlying source mechanism. Two examples of modal AE analysis are presented in (Prosser, 1998). The application to the detection of matrix crack initiation in coupon specimens and delamination detection in rocket motor case rings is demonstrated. According to (McCrory et al., 2015), Measured Amplitude Ratio (MAR) provides improved clarity in the $\mathrm{AE}$ data interpretation compared to unsupervised data-driven techniques. Based on this idea, (Martínez-Jequier, Gallego, Suárez, Juanes, \& Valea, 2015) implemented hardware filters for real-time monitoring of delamination in composite plates. Similarly, (Dahmene et al., 2016) used narrow-band filters for mode separation to extract the dominant mode from AE measurements. Recently, (Barroso-Romero, Gagar, Pant, \& Martinez, 2019) suggested mode identification based on phase and instantaneous frequency using Hilbert transform.

However, despite promising results, the source-sensor distance is identified as major limiting factor for AE-based damage characterization due to frequency dependent attenuation of AE (Maillet, Baker, Morscher, Pujar, \& Lemanski, 2015). In contrast to surface waves (i.e. Rayleigh waves), Lamb waves show frequency dependent amplitude attenuation due to dispersion. Regarding the effect on classical AE parameters, a location-based correction scheme of waveform parameters is implemented by (Aggelis \& Matikas, 2012) using a numerical technique. In an experimental study of $\mathrm{AE}$ due to mechanical loading of composite coupon specimens, (Maillet et al., 2015) reported decrease of frequency centroid with propagation distance. As a solution, a calibration chart for parameter correction depending on propagation distance is suggested. Furthermore, experimental results of AE attenuation in composite plates are presented in (Asamene, Hudson, \& Sundaresan, 2015). A parametric attenuation model is presented taking geometric spreading and material damping into account. According to the results, strong attenuation of the $A_{0}$ mode is observed compared to $S_{0}$ mode. An experimental study regarding the complex propagation behavior of $\mathrm{AE}$ in a rotor system is presented by $(\mathrm{Li}, \mathrm{Lu}, \& \mathrm{Xiao}, 2019)$. Through the literature review it becomes clear that empirical correlations between micro-mechanical damage mechanisms and $\mathrm{AE}$ waveform parameters are useful to characterize different source mechanisms. Promising results are reported using different techniques. However, the application to composite materials is currently limited by the source-sensor distance due to frequency dependent attenuation of AE waveforms. In this paper, new experimental results of acoustic emission attenuation in composite plates are presented. Particular focus is placed on the frequency dependence of acoustic emission attenuation and the effect of different loading conditions. Furthermore, full waveform data are analyzed in time and timefrequency domain using wavelet transform.

The remainder of this paper is structured as follows. In Section 2 experimental procedures are described. New experimental results are presented in Section 3 and implications for AE-based health monitoring are discussed. Finally, summary and conclusions are given in Section 4.

\section{EXPERIMENTS}

In this paper, attenuation of $\mathrm{AE}$ due to wave propagation in Carbon Fiber Reinforced Polymer (CFRP) material is investigated experimentally. As typical specimens, thin CFRP plates with the dimensions $440 \times 440 \times 2 \mathrm{~mm}^{3}$ are used. The specimens are manufactured from CFRP material, which is provided by a major aerospace company as example specimen. Wide specimen dimensions are chosen to enable reflection free measurements of AE waveforms. The experimental setup is illustrated in Figure 1. For artificial source generation and signal acquisition, four disc-shaped Piezoelectric Wafer Active Sensors (PWAS) with $0.55 \mathrm{~mm}$ in thickness and diameter of $\varnothing 10 \mathrm{~mm}$ are used. The PWAS are bonded to the surface of the specimen using cyanoacrylic adhesive. Sensor locations are chosen in the center of the plate at uniform distances of $40 \mathrm{~mm}$. 


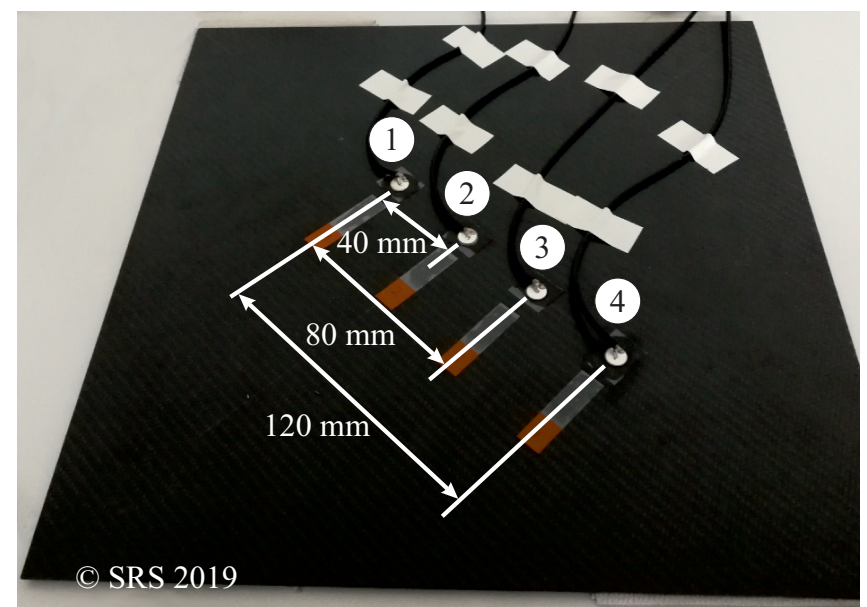

Figure 1. Experimental setup: CFRP plate with four PWAS bonded to the surface.

\subsection{Artificial source generation}

For testing purposes, artificial AE sources are typically used e.g. to verify suitable sensor coupling or to calibrate source localization systems. In literature, the pencil lead break test (PLB), which refers to the excitation of stress waves by manually breaking a pencil lead at the surface of the specimen, is typically used. Even though PLB signals are fairly reproducible, there are different sources of variability due to handling such as free lead length or angle of the lead (Sause, 2011). Alternatively, according to (Kharrat, Placet, Ramasso, \& Boubakar, 2016), repeatable artificial AE sources can be generated using a surface bonded PWAS transmitter as well.

For the intended measurements of AE attenuation, highly reproducible excitations are required to obtain comparable results from multiple experiments. Therefore, artificial AE sources are generated using a surface-bonded PWAS as transmitter to achieve reproducible results. Furthermore, this approach offers well defined excitation. The source characteristics can be easily modified. To simulate AE, the PWAS in location 1 is driven by a signal generator using sine bursts of three cycles at different frequencies.

\subsection{Data acquisition}

The remaining three PWAS transducers at locations 2-4 are used as sensors to record AE waveforms at different distances from the source. For data acquisition, a custom three-channel AE system is used. An impedance transformer is used for signal conditioning before digitization. Measurements are triggered externally and taken in streaming mode to ensure that full waveform data are acquired over the relevant measurement period. The waveform data are sampled continuously at $4 \mathrm{MHz}$ with 16 bit resolution using a dedicated FPGA-based ADC board and stored to a hard drive for subsequent analyses.

\section{RESULTS AND DISCUSSION}

In this section, experimental results regarding the attenuation of simulated AE in CFRP material are presented. First, the attenuation effect is studied qualitatively in time- and timefrequency domain. Subsequently, attenuation coefficients are determined for different frequencies. Finally, the impact of external load is addressed.

\subsection{Effect of propagation on $\mathrm{AE}$ waveforms}

To illustrate the effect of attenuation due to wave propagation qualitatively, measurements of $\mathrm{AE}$ waveforms at distances of $d=40 \mathrm{~mm}, d=80 \mathrm{~mm}$, and $d=120 \mathrm{~mm}$ from the source are presented. As an example, a sine burst comprising three cycles at $100 \mathrm{kHz}$ is chosen as artificial source. In Figure 2, the related AE waveforms recorded at each sensor location are shown in time domain. The time delay between the measurements is attributed to the time-of-flight of the wave packet. From the results, a decrease in amplitude as well as the spreading of the wave packet in time is clearly visible. The cycle number of the wave packet increases with propagation distance, which is related to the dispersion effect. Furthermore, separation of modes due to the different propagation velocities can be observed after a distance of $120 \mathrm{~mm}$.

Similarly, time-frequency domain representation of the AE waveforms is shown in Figure 3. Here, different frequencies are excited by the source which can be detected at sensor 1 after $40 \mathrm{~mm}$ of propagation distance. The peak frequency of the sensor response is detected at $100 \mathrm{kHz}$, which corresponds to the simulated AE source. A second peak is observed above $200 \mathrm{kHz}$. The effect of waveform dispersion is also apparent in this representation. Due to different propagation velocities, the peak amplitude of the higher frequency mode is observed earlier in time than the $100 \mathrm{kHz}$ peak. This effect is more pronounced after a propagation distance of $80 \mathrm{~mm}$. However, whereas the peak frequency of $100 \mathrm{kHz}$ can still be detected after $120 \mathrm{~mm}$ propagation distance, higher frequencies are strongly attenuated and can hardly be detected.

\subsection{Frequency dependence of attenuation}

Subsequently, the frequency dependence of the AE attenuation is investigated. To this end, attenuation coefficients are determined experimentally. In a plate, the relation between initial amplitude of a stress wave $V_{0}$ and the amplitude of a signal $V_{i}$ measured in a propagation distance $x_{i}$ from the source can be assumed as (Asamene et al., 2015)

$$
V_{i}=\frac{1}{\sqrt{x_{i}}} V_{0} \mathrm{e}^{-\alpha x_{i}},
$$

where $\frac{1}{\sqrt{x_{i}}}$ describes the reduction in amplitude due to geometric spreading and exponential decay using attenuation coefficient $\alpha$ describing attenuation due to material damp- 

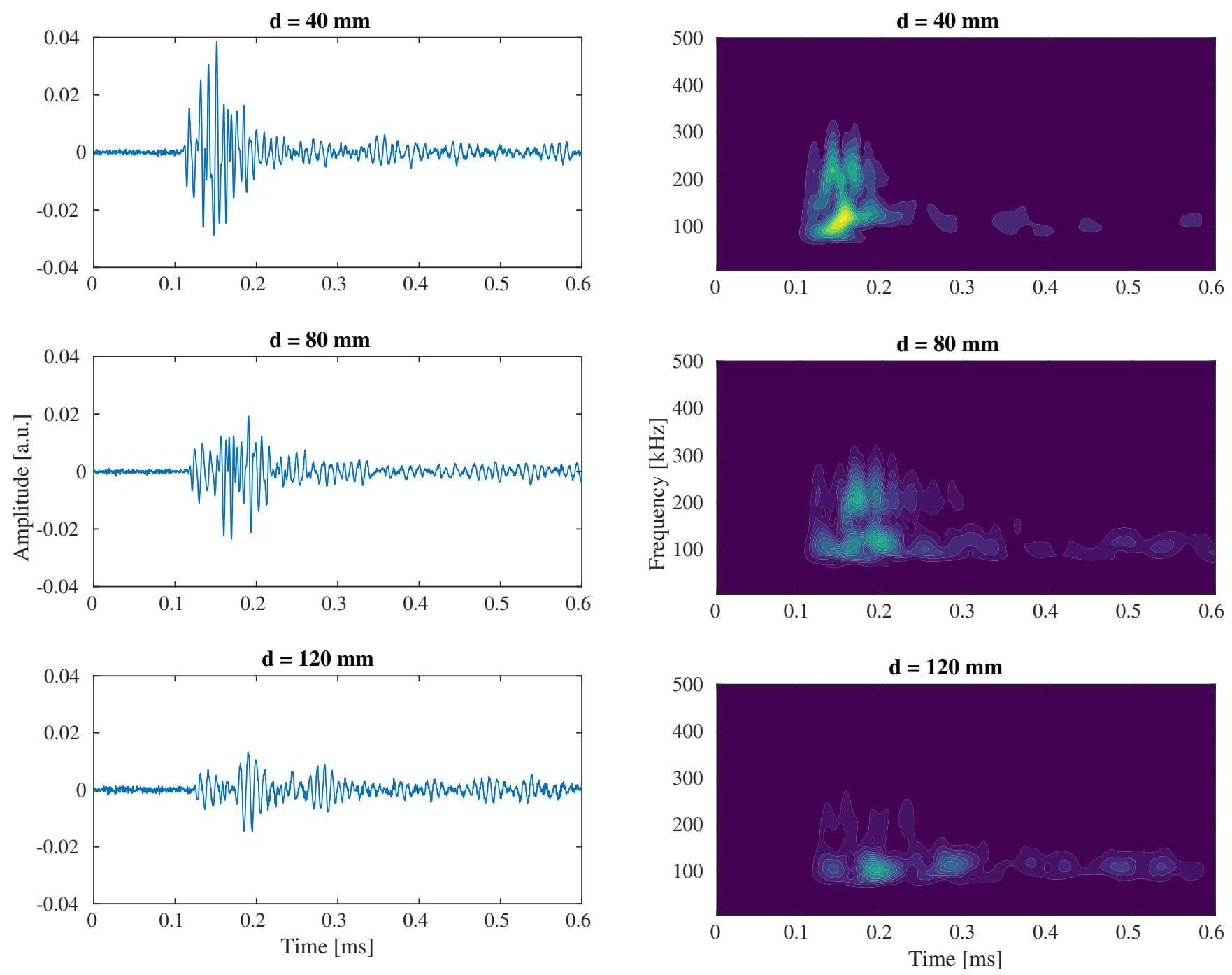

Figure 2. Representative measurements of simulated AE at different distances from source in time domain.

ing. Hence, the damping coefficient $\alpha$ can be calculated as (Asamene et al., 2015)

$$
\alpha=\frac{1}{x_{3}-x_{1}} \ln \left(\frac{V_{1} \sqrt{x_{1}}}{V_{3} \sqrt{x_{3}}}\right)
$$

using the peak amplitudes $V_{1}$ and $V_{3}$ measured at the locations $x_{1}$ and $x_{3}$, which correspond to the propagation distance of $40 \mathrm{~mm}$ and $120 \mathrm{~mm}$, respectively.

In Figure 4, measured peak amplitudes and the theoretical damping behavior are compared at different frequencies of the simulated AE. Here, the theoretical attenuation (solid line) is calculated using damping coefficient $\alpha$, which is determined according to equation 2 . Good agreement between the measurements and theoretical attenuation is observed. Furthermore, it is apparent that attenuation due to material damping is stronger at higher frequency of $280 \mathrm{kHz}$.

Furthermore, frequency dependence is investigated in detail. The frequency of the simulated $\mathrm{AE}$ source is increased from $30 \mathrm{kHz}$ up to $300 \mathrm{kHz}$ in $10 \mathrm{kHz}$ steps and the corresponding attenuation coefficient is determined according to Eq. (2). Each experiment is repeated 100 times. The signals are averaged in time domain to increase signal to noise ratio. Damping coefficients are determined using the root mean square of the related wavelet coefficients.

From the results presented in Figure 5, it can be detected that $\mathrm{AE}$ attenuation varies notably with the frequency of the simulated source. Maximum attenuation of simulated AE waveforms is obtained at a frequency of $150 \mathrm{kHz}$. Increasing attenuation coefficients are obtained at frequencies from $50 \mathrm{kHz}$ up to $150 \mathrm{kHz}$. Above $150 \mathrm{kHz}$ attenuation decreases at first leading to lower attenuation between $200 \mathrm{kHz}$ and $250 \mathrm{kHz}$. However, above $250 \mathrm{kHz}$ increasing attenuation of simulated 


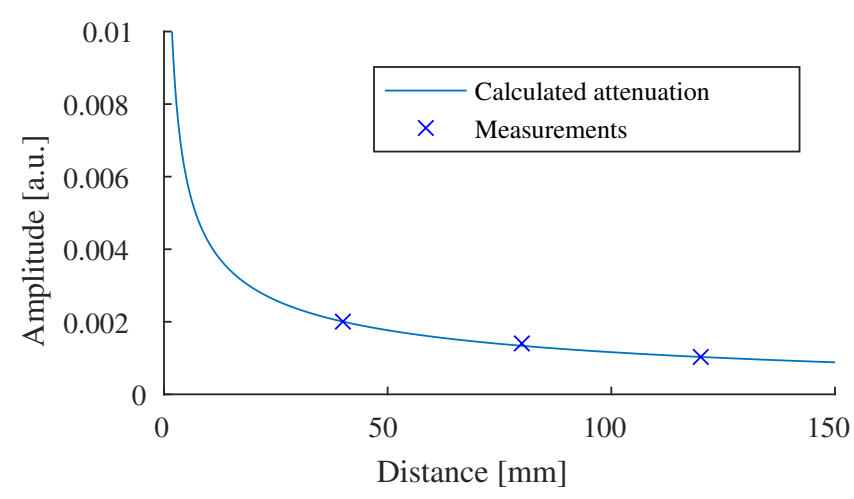

(a) $60 \mathrm{kHz}$

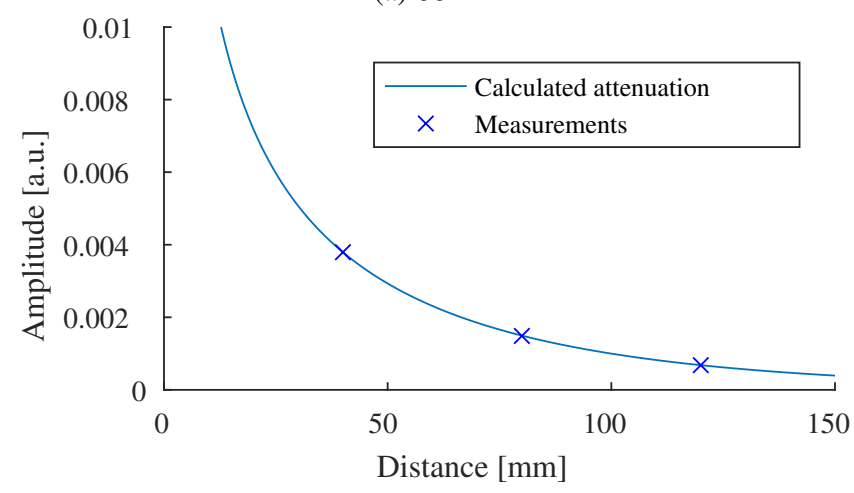

(b) $280 \mathrm{kHz}$

Figure 4. Comparison of theoretical AE attenuation and measured peak amplitudes.

AE is observed again. Therefore, it can be concluded that for a specific transfer path between source and sensor, detectability of an $\mathrm{AE}$ source depends also on the frequency spectrum of the corresponding source mechanism. Furthermore, it can be expected that due to dispersion and attenuation, AE signatures of different source mechanisms in frequency domain are not invariant to the propagation path. In particular, regarding data-driven approaches based on statistical pattern recognition, which are widely studied for source characterization of $\mathrm{AE}$, it has to be noted that dispersion and frequency dependent attenuation could have an impact on the distinguishability of different source mechanisms.

\subsection{Effect of loading conditions}

Finally, experimental results regarding the effect of different loading conditions on the attenuation of simulated $\mathrm{AE}$ are presented. External bending load is applied using a clamping mechanism as illustrated in Figure 6. Here, the load is applied by clamps attached to the free edges and a support placed at the center of the plate. A total of five different loads is considered by increasing the vertical displacement of the edges in $10 \mathrm{~mm}$ steps.

Similar to Section 3.2, attenuation coefficients are determined for simulated $\mathrm{AE}$ sources with frequencies between $30 \mathrm{kHz}$

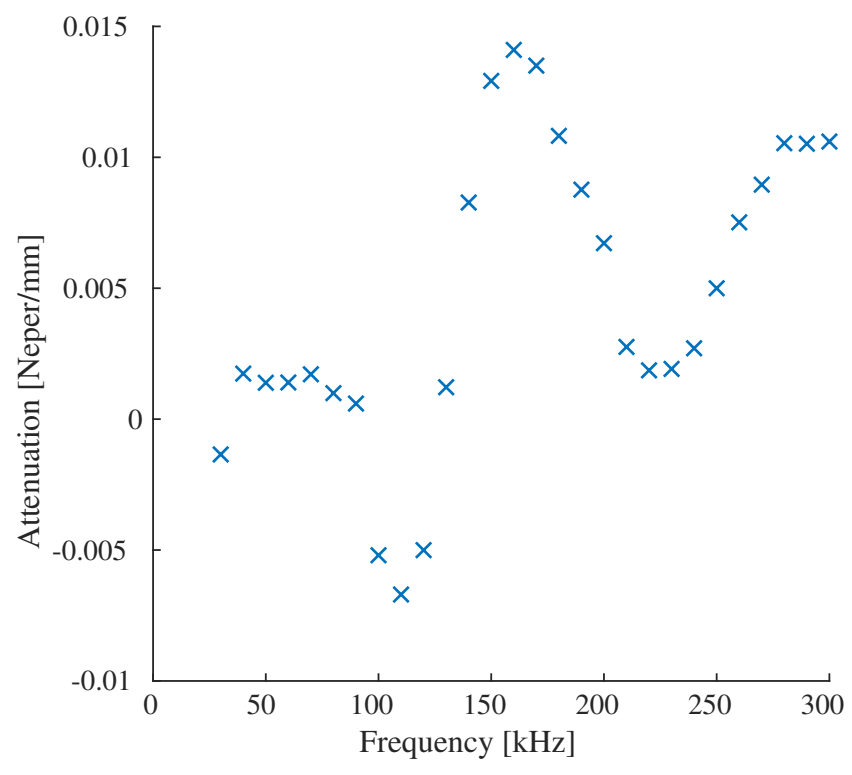

Figure 5. Attenuation coefficients determined at different frequencies of the simulated $\mathrm{AE}$ source.

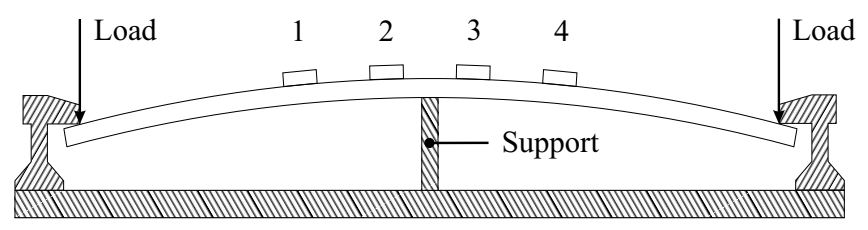

Figure 6. Schematic illustration of the clamping mechanism.

and $300 \mathrm{kHz}$. The results are shown in Figure 7. Here, it is notable that in this experiment slight changes in external loading conditions have already a strong impact on the attenuation characteristics of the specimen. Compared to the unloaded case, stronger attenuation of the $\mathrm{AE}$ waveforms is observed over a wide range of excitation frequencies if external load is applied. This effect is particularly pronounced below $100 \mathrm{kHz}$ and above $150 \mathrm{kHz}$. In contrast to the unloaded case, where the attenuation coefficients decrease at frequencies from $150 \mathrm{kHz}$ to $200 \mathrm{kHz}$, increasing attenuation coefficients are obtained in this frequency range. Furthermore, maximum attenuation is shifted to approximately $250 \mathrm{kHz}$ in case of the loaded specimen. Above $250 \mathrm{kHz}, \mathrm{AE}$ attenuation shows a decreasing trend. According to these results it can be concluded that to achieve robust detection and classification of $\mathrm{AE}$, loading conditions have to be taken into consideration.

\section{SUMMARY AND CONCLUSIONS}

In this paper, new experimental results regarding the $\mathrm{AE}$ attenuation characteristics of CFRP material due to wave propagation are presented. As typical specimens, thin plates manufactured from aerospace material are used. From the results it becomes clear that attenuation of AE due to propagation increases with frequency. Furthermore, increasing attenuation 


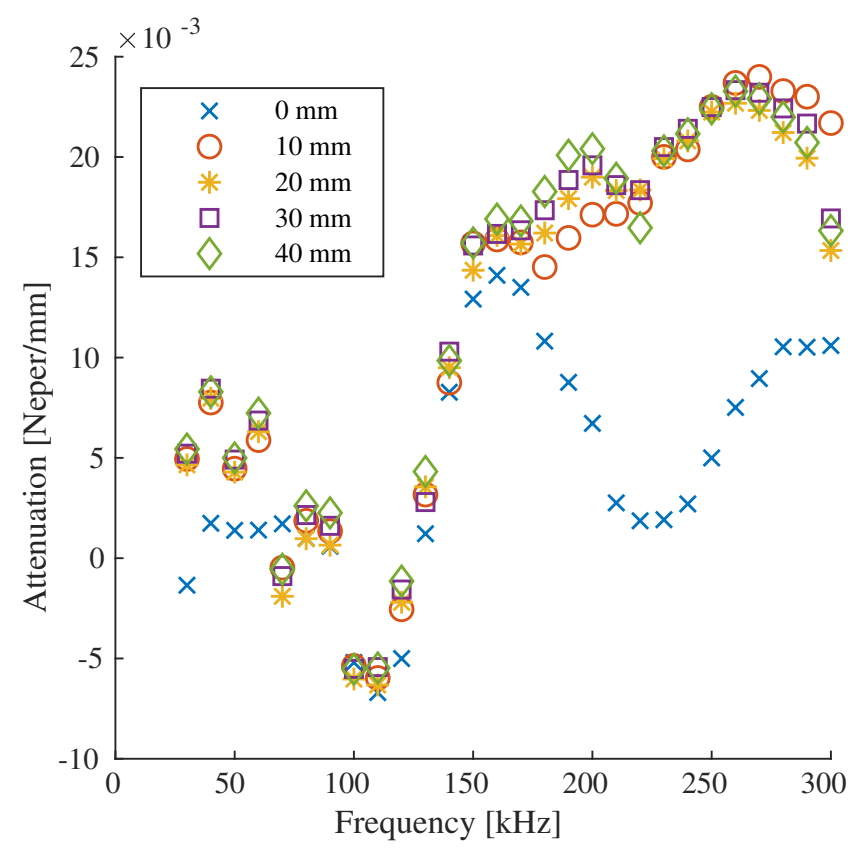

Figure 7. Comparison between attenuation coefficients of free and clamped plate.

of the simulated $\mathrm{AE}$ waveforms is observed due to external bending load. Therefore, it can be concluded that source frequency and external load have an impact on the detectability of AE. To improve AE data analyses and interpretation, the impact of wave propagation and different loading conditions on the detectability of different source mechanisms has to be considered. Consequently, this includes the impact of attenuation on the performance of data-driven classification approaches.

\section{ACKNOWLEDGEMENT}

The authors are thankful for the CFRP specimens kindly provided by major aerospace company.

\section{REFERENCES}

Aggelis, D. G., \& Matikas, T. E. (2012). Effect of plate wave dispersion on the acoustic emission parameters in metals. Computers and Structures, 98-99, 17-22.

Asamene, K., Hudson, L., \& Sundaresan, M. (2015). Influence of attenuation on acoustic emission signals in carbon fiber reinforced polymer panels. Ultrasonics, 59, 86-93.

Baccar, D., \& Söffker, D. (2017). Identification and classification of failure modes in laminated composites by using a multivariate statistical analysis of wavelet coefficients. Mechanical Systems and Signal Processing, 96, 77-87.

Barroso-Romero, M., Gagar, D., Pant, S., \& Martinez, M.
(2019). Wave Mode Identification of Acoustic Emission Signals Using Phase Analysis. Acoustics, 1(2), 450-472.

Beheshtizadeh, N., Mostafapour, A., \& Davoodi, S. (2019). Three point bending test of glass/epoxy composite health monitoring by acoustic emission. Alexandria Engineering Journal, in press.

Crivelli, D., Guagliano, M., Eaton, M., Pearson, M., AlJumaili, S., Holford, K., \& Pullin, R. (2015). Localisation and identification of fatigue matrix cracking and delamination in a carbon fibre panel by acoustic emission. Composites Part B: Engineering, 74, 1-12.

Dahmene, F., Yaacoubi, S., El Mountassir, M., Bendaoud, N., Langlois, C., \& Bardoux, O. (2016). On the modal acoustic emission testing of composite structure. Composite Structures, 140, 446-452.

Gutkin, R., Green, C., Vangrattanachai, S., Pinho, S., Robinson, P., \& Curtis, P. (2011). On acoustic emission for failure investigation in CFRP: Pattern recognition and peak frequency analyses. Mechanical Systems and Signal Processing, 25(4), 1393-1407.

Hamdi, S. E., Le Duff, A., Simon, L., Plantier, G., Sourice, A., \& Feuilloy, M. (2013). Acoustic emission pattern recognition approach based on Hilbert-Huang transform for structural health monitoring in polymercomposite materials. Applied Acoustics, 74(5), 746757.

Johnson, M., \& Gudmundson, P. (2000). Broad-band transient recording and characterization of acoustic emission events in composite laminates. Composites Science and Technology, 60(15), 2803-2818.

Kharrat, M., Placet, V., Ramasso, E., \& Boubakar, M. L. (2016). Influence of damage accumulation under fatigue loading on the AE-based health assessment of composite materials: Wave distortion and AE-features evolution as a function of damage level. Composites Part A: Applied Science and Manufacturing, 109, 615627.

Li, Z., Lu, W., \& Xiao, Y. (2019). Propagation characteristics of acoustic emission signal in rotor system. Advances in Engineering Research, 184, 189-193.

Maillet, E., Baker, C., Morscher, G. N., Pujar, V. V., \& Lemanski, J. R. (2015). Feasibility and limitations of damage identification in composite materials using acoustic emission. Composites Part A: Applied Science and Manufacturing, 75, 77-83.

Marec, a., Thomas, J. H., \& El Guerjouma, R. (2008). Damage characterization of polymer-based composite materials: Multivariable analysis and wavelet transform for clustering acoustic emission data. Mechanical Systems and Signal Processing, 22(6), 1441-1464.

Martínez-Jequier, J., Gallego, A., Suárez, E., Juanes, F. J., \& Valea, Á. (2015). Real-time damage mechanisms assessment in CFRP samples via acoustic emission Lamb 
wave modal analysis. Composites Part B: Engineering, 68, 317-326.

McCrory, J. P., Al-Jumaili, S. K., Crivelli, D., Pearson, M. R., Eaton, M. J., Featherston, C. A., ... Pullin, R. (2015). Damage classification in carbon fibre composites using acoustic emission: A comparison of three techniques. Composites Part B: Engineering, 68, 424-430.

Nazmdar Shahri, M., Yousefi, J., Fotouhi, M., \& Ahmadi Najfabadi, M. (2016). Damage evaluation of composite materials using acoustic emission features and Hilbert transform. Journal of Composite Materials, 50(14), 1897-1907.

Ni, Q. Q., \& Iwamoto, M. (2002). Wavelet transform of acoustic emission signals in failure of model composites. Engineering Fracture Mechanics, 69(6), 717728.

Prosser, W. H. (1998). Waveform analysis of AE in composites. In Proceedings of the sixth international symposium on acoustic emission from composite materials (pp. 61-70). San Antonio.

Sause, M. G. R. (2011). Investigation of pencil-lead breaks as acoustic emission sources. Journal of Acoustic Emission, 29, 184-196.

Wirtz, S. F., Beganovic, N., \& Söffker, D. (2019). Investigation of damage detectability in composites using frequency-based classification of Acoustic Emission measurements. Structural Health Monitoring, 18(4), 1207-1218.

\section{BIOGRAPHIES}

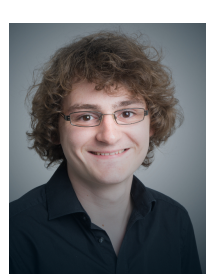

Sebastian Felix Wirtz received both B.Sc. and M.Sc. degree in mechanical engineering with an emphasis on mechatronics in 2014 and 2015 from University of Duisburg Essen, Germany. From 2013 until 2015 he was working as student assistant in the Chair of Dynamics and Control, University of Duisburg-Essen, Germany, where he is currently pursuing his $\mathrm{Ph} . \mathrm{D}$. degree in the area of structural health monitoring. His research interests include acoustic emission, diagnosis, and machine learning.

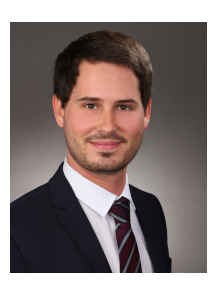

Stefan Bach received the B.Sc. degree in electrical engineering from the University of Duisburg Essen, Germany in 2016. Currently he is working on his master thesis in the field of structural health monitoring. His research interests include signal processing, fault diagnosis, and embedded systems.

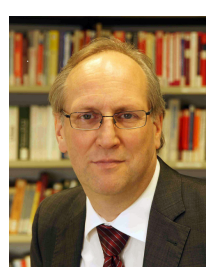

Dirk Söffker received the Dr.-Ing. degree in mechanical engineering and the Habilitation degree in automatic control/safety engineering from University of Wuppertal, Germany, in 1995 and 2001, respectively. Since 2001, he leads the Chair of Dynamics and Control, University of Duisburg-Essen, Germany. His current research interests include diagnostics and prognostics, modern methods of control theory, human interaction with safe human interaction with technical systems, safety and reliability control engineering of technical systems, and cognitive technical systems. 